\title{
Extreme-Ultraviolet Vortices at a Free-Electron Laser
}

Primož Rebernik Ribič, ${ }^{1}$ Benedikt Rösner, ${ }^{2 *}$ David Gauthier, ${ }^{1}$ Florian Döring, ${ }^{2}$ Claudio Masciovecchio, ${ }^{1}$ Emiliano Principi, ${ }^{1}$ Christian David, ${ }^{2}$ Giovanni De Ninno ${ }^{1,3}$

${ }^{1}$ Elettra-Sincrotrone Trieste, Strada Statale 14-km 163,5, 34149 Basovizza, Trieste, Italy

${ }^{2}$ Paul Scherrer Institut, 5232 Villigen PSI, Switzerland

${ }^{3}$ Laboratory of Quantum Optics, University of Nova Gorica, 5001 Nova Gorica, Slovenia

* Benedikt Rösner, benedikt.roesner@psi.ch

Optical vortices are helically phased light beams with a field dependence of $\exp (i l \phi)$, where $l$ is the topological charge and $\phi$ the azimuthal coordinate in the plane perpendicular to the beam propagation. Such beams carry an orbital angular momentum (OAM) of $l \hbar$ per photon. The interaction between matter and extreme-ultraviolet (EUV) optical vortices is predicted to trigger new phenomena. Among these are violation of dipolar selection rules during photoionization [1], generation of charge current loops in fullerenes with an associated orbital magnetic moment [2], and the production of skyrmionic defects [3], promising for applications magnetic memory devices on the nanoscale.

An elegant method to realize optical vortices with X-rays is the use of a spiral zone plate (SZP) [4] similar to a Fresnel zone plate. This optical element produces a focused vortex without extensive modifications of the optics upstream. Implemented at the FERMI free electron laser (FEL), the optical vortex can be obtained directly from the fundamental (transversely Gaussian) FEL beam in the EUV regime. The resulting EUV beam exhibits an azimuthal phase shift, a torus-shaped focal spot (see Figure 1 ), and can produce power densities at the FERMI FEL of up to $10^{17} \mathrm{~W} / \mathrm{cm}^{2}[5]$.

The possibility to produce optical vortices in the X-ray regime using SZPs was demonstrated with synchrotron radiation [4]. The high photon flux at an FEL, however, imposes serious limitations for the design of such zone plates, as many common materials cannot withstand even the unfocused FEL beam intensity [6]. An excellent material for the fabrication of radiation-hard diffractive optical elements in the XUV regime is silicon [7]. The SZPs used in the experiment were etched into ultra-flat, $340 \mathrm{~nm}$ thick silicone membranes purchased from Norcada, Inc. (Canada). For this purpose, the silicon membrane was coated with a chromium layer and a polymer resist, which was subsequently exposed with the zone plate pattern using e-beam lithography. After development, the pattern was transferred into the chromium layer by reactive-ion etching (RIE), which was then used as a hard mask in a subsequent RIE step to etch the zone plates 200-230 nm deep into the silicon membrane. The SZPs were designed for a wavelength of $26 \mathrm{~nm}$ and had a focal length of $120 \mathrm{~mm}$. The SZP diameter was $1.90 \mathrm{~mm}$, with an outermost zone width of $1.64 \mu \mathrm{m}$. The lateral dimensions of opaque and transparent zones were adjusted to a ratio of 60:40. This geometry almost completely suppresses the $0^{\text {th }}$ diffraction order (the directly transmitted FEL beam) that carries no OAM without the use of a central beam stop. The phase of the radiation focused by conventional Fresnel zone plates (FZP) and spiral zone plates with several topological charges $l$ was recorded in the far field using a Hartmann wavefront sensor [8], whereas the intensity distributions in focus were reconstructed from developed polymer imprints [9].

The standard Fresnel zone plate (FZP, Figure 1a), produces a Gaussian-like beam in the far-field with an almost flat phase profile (after removing the quadratic curvature introduced by pre-focusing). The residual phase contrast is due to the aberrations of the beam alignment mirrors, aberrations in the FEL 
beam itself, and the detection noise at the wavefront sensor. The intensity distribution in the focal plane of the (conventional) FZP shows its typical diffraction pattern. The focal spot of the Fresnel zone plate can be described with the first-order Bessel function and a Rayleigh spot size of $2.0 \mu \mathrm{m}$, in exact agreement with theory.
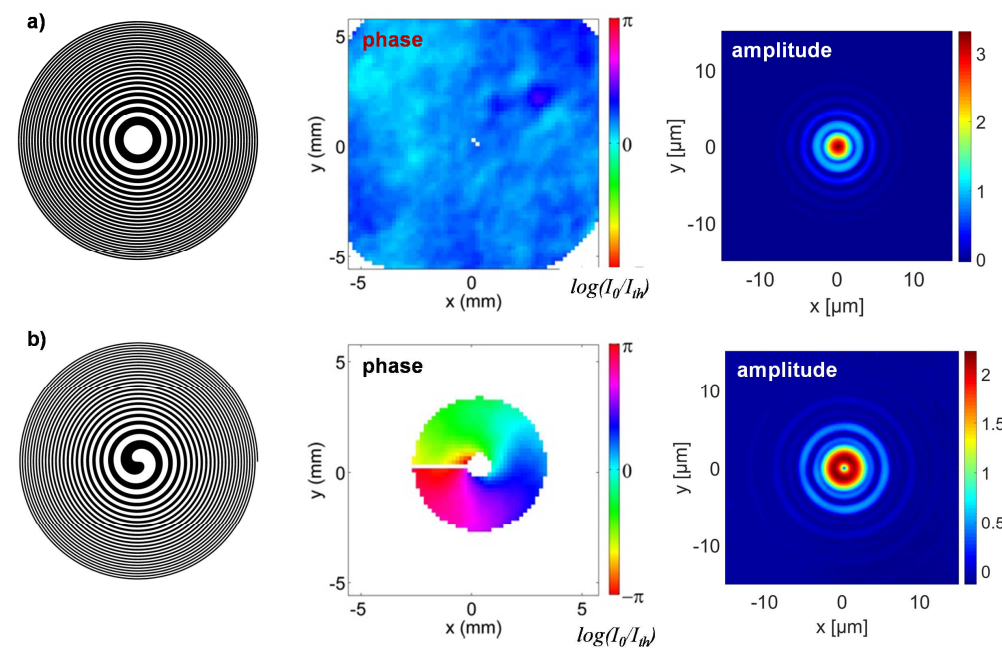

Figure. 1. a) Zone plate pattern, resulting phase and amplitude of a conventional Fresnel zone plate. b) Zone plate pattern, resulting phase and amplitude of a spiral zone plate with $l=1$.
The SZP with a topological charge of one produces a phase profile which is in good agreement with the theory. Exhibiting a singularity in the center, the phase is shifted by $2 \pi$, or one wavelength in $360^{\circ}$ around the optical axis. The focal spot is torus-shaped and can be described using a hypergeometric function [9]. Higher topological charges $l$ result in phase profiles with a phase shift of $|l| \cdot 2 \pi$.

Assuming $17 \%$ efficiency for the SZP and a typical energy per pulse of $10 \mu \mathrm{J}$ (50 fs pulse duration) used during the experiment, a power density on the order of $10^{14} \mathrm{~W} / \mathrm{cm}^{2}$ is estimated in focus. The zone widths, and thus the spot size of the

$\mathrm{ZP}$ in focus, can be easily reduced by a factor of 50-100. In combination with a maximum energy per pulse of $100 \mu \mathrm{J}$, which is routinely achieved at FERMI, the power density can be increased by three to four orders of magnitude.

\section{References:}

[1] Picón A, Benseny A, Mompart J et al, New J Phys 12 (2010), p. 083053.

[2] Wätzel J, Pavlyukh Y, Schäffer A et al, Carbon 99 (2016), p. 439.

[3] Fujita H and Sato M, Phys Rev B 95 (2017), p. 054421.

[4] Vila-Comamala J, Sakdinawat A and Guizar-Sicairos M, Opt Lett 39 (2014), p. 5281.

[5] Ribič P R, Rösner B, Gauthier D et al, Phys Rev X 7 (2017), p. 031036.

[6] David C, Gorelick S, Rutishauser S et al, Sci Rep 1 (2011), p. 57.

[7] Vila-Comamala J, Jefimovs K, Raabe J et al, Microelectron Eng 85 (2008), p. 1241.

[8] Ribič P R, Gauthier D and De Ninno G, Phys Rev Lett 112 (2014), p. 203602.

[9] Rösner B, Döring F, Ribič P R et al, Opt Express 25 (2017), p. 30686. 